\title{
Microstructure and Material Properties of Milk Fat Systems During Temperature Fluctuations
}

\author{
Patrizia Buldo • Ulf Andersen • Lars Wiking
}

Received: 10 January 2013 / Accepted: 30 April 2013 /Published online: 2 June 2013

(C) Springer Science+Business Media New York 2013

\begin{abstract}
Microstructure and material properties of milk fat systems containing different amount of milk fat globules and different emulsion types were studied towards the effects of temperature fluctuations stress. The three systems were (i) an emulsion based on anhydrous milk fat (AMF) consisting of crystals dispersed in liquid oil, (ii) butter which is a water in oil emulsion consisting of a continuous crystal network with partially disrupted fat globules interrupted by intact fat globules and water droplets, and (iii) freeze-dried cream which is an oil in water emulsion consisting of concentrated milk fat globules. The microstructure of the systems was observed by confocal laser scanning microscopy and linked to their material properties by large and small deformation rheology techniques. Different rheological techniques were used to characterize the material descriptors. The crystallization behavior was studied by thermal analysis and pulsed nuclear magnetic resonance. The freeze-dried cream was characterized as the softest, more elastic, and less stiff when compared to butter and AMF. Temperature fluctuations led to drastic material consolidation in butter as a consequence of flocculation of fat globules and formation of a more dense crystal network with stronger bonds. Only after several temperature fluctuations, material consolidation was observed in AMF, whereas no significant changes in material properties were observed in the freeze-dried cream. The intact fat globules decrease the hardness and stiffness, whereas the amount and
\end{abstract}

P. Buldo $\cdot$ L. Wiking $(\bowtie)$

Department of Food Science, Aarhus University,

Blichers Allé 20, P.O. box 50, 8830 Tjele, Denmark

e-mail: Lars.Wiking@agrsci.dk

P. Buldo

e-mail: pbu@life.ku.dk

U. Andersen

Arla Strategic Innovation Centre, Arla Foods,

Rørdrumvej 2, 8220 Brabrand, Denmark type of contact points between crystal clusters of the dispersed crystal phase contribute to increase hardness, stiffness, and brittleness of the systems.

Keywords Emulsion · Milk fat globule $\cdot$ Crystallization · Temperature fluctuations $\cdot$ Rheology

\section{Introduction}

Milk fat in its natural state is present as oil in water $(\mathrm{O} / \mathrm{W})$ emulsion, e.g. in milk and cream, which are composed of milk fat globules surrounded by a biological membrane of phospholipid and proteins dispersed in a water phase. Butter is produced from churning of the cream, where phase inversion of the emulsion occurs, from $\mathrm{O} / \mathrm{W}$ of the cream to water in oil (W/O) emulsion. The microstructure of butter consists of a continuous crystal network dispersed in liquid oil and interrupted by fat globules, water and air droplets. The crystal network is a three-dimensional structure formed by crystals or crystal clusters, which are hold together by primary and secondary bonds $[1,2]$. Primary bonds are strong and irreversible, and they characterize the hardness of the product, whereas, the consistency of the product is mainly define by secondary bonds which are weak and reversible [1-3]. It is generally assumed that the presence of fat globules in the microstructure of butter decreases the hardness of the product, as the continuous fat crystal network is interrupted by the globules [3, 4]. The milk fat globules contain a considerable part of crystalline fat, as during churning of the cream mainly liquid fat is extruded from the milk fat globules. Moreover, the crystal fats from the high melting fraction (HMF) are present inside the partly intact milk fat globules, thus low contribution to the overall strength of the crystalline network is expected. Anhydrous milk fat (AMF) is produced by removal of the milk fat globule membrane as well as the water phase, so that only triacylglycerols (TAGs; $99.8 \%$ ) are present. Despite the 
same fatty acids composition, the crystallization mechanisms of butter and AMF are different [5-7]. In general, in milk fat globules the supercooling needed to start crystallization is higher, the crystallization onset is delayed, and the crystallization rate is lower when compared to bulk fat $[4,5,8]$. Contrary to bulk fat, in milk fat globules crystallization has to occur in every single droplet independently [5], therefore the time needed for crystallization is longer. Handling and storage of milk fat based products may initiate partial melting of the crystal network followed by recrystallization. Many consumers have experienced that taking the butter in and out of the fridge, resulting in partial melting and recrystallization of the fat, often results in a harder and less spreadable product over time. The role of the ratio between the continuous and globular fat phase in this process is not elucidated yet. The objective of this study was to characterize the material properties of different milk fat systems, i.e. partially disrupted milk fat globules, concentrate milk fat globules, and AMF-based emulsion, and to evaluate their stability towards temperature fluctuation stress. For this purpose, cream was freeze-dried in order to produce a system with concentrated milk fat globules. Different geometries among the large deformation rheology tests were used to describe the material properties of the milk fat systems.

\section{Material and Method}

Preparation and Temperature Fluctuation Program of the Milk Fat Systems

An emulsion based on AMF (Arla Foods, Sweden), butter (Arla Foods, Viby J., Denmark), and a system of concentrated milk fat globules were studied. The latter was prepared by freeze drying pasteurized cream with $38 \%$ of fat (Arla Foods, Brabrand, Denmark) with a bench top freeze dryer (Scanvac, LaboGen, Denmark), until a water content of $16 \%$ was reached. The water content of the three systems was always the same. Water content was measured in a HR73 Halogen Moisture Analyzer (Mettler Toledo, Greifensee, Swizzerland). The cream was freeze-dried in batch of 11 and then pooled together, mixed and shaped in rectangular blocks of approximately $250 \mathrm{~g}$. The cream used for freeze drying and AMF, were heated to $65^{\circ} \mathrm{C}$ and kept at this temperature for $15 \mathrm{~min}$, in order to erase the thermal history. The cream was cooled to $10^{\circ} \mathrm{C}$ and kept at this temperature for $1 \mathrm{~h}$ before freeze drying $\left(-108{ }^{\circ} \mathrm{C}\right.$ and $\left.0.633 \mathrm{hPa}\right)$. Whereas, AMF was incubated at $40{ }^{\circ} \mathrm{C}$ for $2 \mathrm{~h}$ before blending it with $16 \%$ of butter milk for 2 min at maximum speed with a table top-blender (Hobartin Offenburg, Germany). Butter, AMF-based emulsion, and freeze-dried cream were stored at $5{ }^{\circ} \mathrm{C}$ for 2 weeks before analysis. Afterwards, the three systems were subjected to a temperature fluctuation program (Table 1). All the measurements were performed the day after temperature fluctuation of the samples. However, in the text we will refer to the day of temperature fluctuation, i.e. day one corresponds to the samples stored at $25^{\circ} \mathrm{C}$ for $30 \mathrm{~min}$ and consequently stored at refrigerator temperature for approximately $24 \mathrm{~h}$ before analysis. The temperature fluctuation program was designed to study the effect of temperature cycling on the different milk fat systems (from day 1 to day 3), moreover, the effect of a prolonged exposure time to $25^{\circ} \mathrm{C}$ was also studied (day 4 ), as well as the effect that the refrigerator temperature has on the system after temperature fluctuations (day 5 and day 8).

\section{Melting Profile of the Milk Fat Systems}

The melting profile of the three systems was analyzed before temperature fluctuation (day zero) and at the end of the temperature fluctuation (day 8) by a Differential Scanning Calorimetry (DSC, Q2000 TA Instrument, UK). From 10 to $15 \mathrm{mg}$ of sample was loaded in a hermetically sealed aluminum pan. The reference used was an empty sealed pan. The samples were equilibrated at $5{ }^{\circ} \mathrm{C}$ for $5 \mathrm{~min}$. Afterwards, the temperature was increased to $60{ }^{\circ} \mathrm{C}$ at a rate of $5{ }^{\circ} \mathrm{C} / \mathrm{min}$. Peak minima of the endothermic curves were used. DSC analyses were performed at least in triplicate.

\section{Solid Fat Content Determination of the Milk Fat Systems}

Solid fat content (SFC) of the milk fat systems was measured by a low field pulsed Nuclear Magnetic Resonance Spectrometer (NMR, Resonance Maran Ultra, UK) by direct method (AOCS Cd 16b-93). The samples were equilibrated at $5{ }^{\circ} \mathrm{C}$ before performing the analysis. The analyses were run in quadruplicate.

\section{Oscillation Analyses of the Milk Fat Systems}

Oscillatory experiments were performed on a rheomether (AR-G2, TA Instrument, US based) using $40 \mathrm{~mm}$ parallel plate geometry. Temperature control $\left(5^{\circ} \mathrm{C} \pm 0.1{ }^{\circ} \mathrm{C}\right)$ was achieved by a Peltier element. The linear viscoelastic region (LVR) was determined for every system by performing an oscillatory strain sweep test from $1.00 \mathrm{E}-4 \%$ to $100 \%$ with a constant frequency of $1 \mathrm{~Hz}$. A frequency sweep test was performed from 0.1 to $100 \mathrm{~Hz}$, with a constant strain (5.04E-3 \%). For each system, from five to nine repetitions were performed. Rheograms were obtained by plotting the elastic moduli, $\mathrm{G}^{\prime}(\mathrm{Pa})$, as a function of the angular frequency $(\mathrm{rad} / \mathrm{s})$.

Material Properties of the Milk Fat Systems

Large deformation rheology was performed by Texture Analyser (TA-HDi, Stable Micro System Ltd., Surrey, England) 
Table 1 Temperature fluctuation program used in the study. When not incubated at $25{ }^{\circ} \mathrm{C}$, the sample was stored at $5{ }^{\circ} \mathrm{C}$

\begin{tabular}{|c|c|c|c|c|c|c|c|}
\hline Day1 & Day2 & Day3 & Day4 & Day5 & Day6 & Day7 & Day8 \\
\hline $25^{\circ} \mathrm{C}$ for $30 \mathrm{~min}$ & $25^{\circ} \mathrm{C}$ for $30 \mathrm{~min}$ & $25^{\circ} \mathrm{C}$ for $30 \mathrm{~min}$ & $25^{\circ} \mathrm{C}$ for $1.5 \mathrm{~h}$ & No fluctuation & $25^{\circ} \mathrm{C}$ for $30 \mathrm{~min}$ & No fluctuation & No fluctuation \\
\hline
\end{tabular}

with a $100 \mathrm{Kg}$ load cell and $0.001 \mathrm{~N}$ detecting range. Different geometries were used: perspex $30^{\circ}$ conical geometry; $2 \mathrm{~mm}$ stainless needle; $0.5 \mathrm{~cm}^{2}$ delrin cylinder and $75 \mathrm{~mm}$ aluminum parallel plate. Penetration tests were performed by using a penetration speed of $0.2 \mathrm{~mm} / \mathrm{s}$ and a distance of $8 \mathrm{~mm}$. While, uniaxial compression analyses were performed at a speed of $1.2 \mathrm{~mm} / \mathrm{s}$ and a distance of $9 \mathrm{~mm}$. For the compression test, disks of $11.7 \pm 1 \mathrm{~mm}$ height and $24 \mathrm{~mm}$ diameter were used.

The maximal force at the defined penetration distance, as recorded from the penetration test performed by needle and cone geometries, was used as indicator for the relative hardness of the system. Whereas, the force at the breaking point, as illustrated by the force-distance curve obtained by cylinder geometry, was used as indicator of true hardness of the system. The material properties of the systems was further evaluated by converting the force $(\mathrm{N})$ and displacement $(\mathrm{mm})$ at the breaking point, obtained by compression test and penetration test with a cylinder, into true axial stress, $\sigma(\mathrm{Pa})(\mathrm{Eq} .1)$ :

$\sigma=\frac{F}{A} \frac{H}{H i}[\mathrm{~Pa}]$

where $\mathrm{F}=$ force $(\mathrm{N}), \mathrm{A}=$ initial cross-sectional area $\left(\mathrm{m}^{2}\right), \mathrm{H}_{i}=$ initial sample height $(\mathrm{mm})$, and $\mathrm{H}=$ height $(\mathrm{mm})$. Moreover, from the force-distance curves obtained by compression test the maximum slope, in a range of $0.5 \mathrm{~mm}$, was calculated as indicator of the elasticity of the systems. Brittleness was evaluated by the presence of irregularities showed after the breaking point on the force-distance curves, obtained by compression test and penetration test performed by cylinder geometry. All tests were performed at room temperature $\left(20 \pm 1^{\circ} \mathrm{C}\right)$ and forces were registered with a standard Instron recorder having a response time of about $1 \mathrm{~s}$. At least five replicates of each sample were analyzed in the compression test, whereas a minimum of three samples were analyzed in the penetration tests.

\section{Microstructure Analyses of the Milk Fat Systems}

The microstructure of the three systems was analyzed by an inverted confocal laser scanning microscopy (CLSM; Leica TCS SP2, Germany), as previously described [9, 10]. Nile red (Sigma Aldrich, St. Louis, MO), FITC (Merck, Darmstadt, Germany), and Rhodamine (Avanti Polar Lipids, Inc., AL, USA) were used as the fluorescence stain agents for fats, proteins, and milk fat globule membrane, respectively. Nile red and FITC were dissolved in acetone to a final concentration of $0.01 \%$, whereas Rhodamine $(1 \mathrm{mg} / \mathrm{mL})$ was dissolved in chloroform to a final concentration of $0.01 \%$. The dye solutions were added to a pre-cooled $\left(4{ }^{\circ} \mathrm{C}\right)$ glass slide and after that the solvent evaporated, sample was added. The prepared slides were stored at $4{ }^{\circ} \mathrm{C}$, for approximately $30 \mathrm{~min}$, before microstructure analysis. Argon $488 \mathrm{~nm}$ and $\mathrm{HeNe} 561 \mathrm{~nm}$ laser were used for excitation to induce fluorescence emission. A $60 \mathrm{X}$ water-immersion objective was used. The images were recorded at a speed of $400 \mathrm{~Hz}$ and 8-bit resolution.

On the images obtained from butter and freeze-dried cream stained with Rhodamine, image analysis was performed with ImageJ (available at http://rsb.info.nih.gov/ij; developed by Wayne Rasband, National Institutes of Health, Bethesda, MD). For each sample, from 10 to 24 images were analyzed, and the average of the mean intensity value was calculated. The pixel intensity was quantified by transforming the RGB images into single channel and then by converting them to greyscale. Measurements were carried out on binary images.

\section{Statistical Analysis}

The data obtained by DSC, NMR and by small and large deformation rheology tests were statistically analyzed by two-way analysis of variance, followed by Tukey's multiple comparison test ( $\mathrm{R}$, version 2.15.0; www.r-project.org). The data obtained by image analysis were statistically analyzed by one-way analysis of variance, followed by Tukey's multiple comparison test (R, version 2.15.0; www.r-project.org). All data are reported as average plus/minus standard error.

\section{Results}

Microstructure and Material Properties of the Milk Fat Systems Before Temperature Fluctuations

The microstructure of the three milk fat systems was very different among them, as observed by CLSM (Fig. 1a, b and c). The microstructure of butter was characterized by a continuous fat crystal network (black shadow) immersed in a continuous oil phase (illustrated by the brownish color given by the Nile red dye) together with water/protein and air droplets (green and black droplets, respectively) (Fig. 1a). Moreover, intact milk fat globules can also be distinguished in the images (arrows in Figs. 1a and 2a). The microstructure of freeze-dried cream was characterized by a continuous water and protein phase (illustrated by the yellowish color given by the FITC dye) with intact fat globules as dispersed phase (Figs. 1b and 2b). Neither crystals, nor crystal 
Fig. 1 Confocal laser scanning microscopy images at day 0 for butter (a), freeze-dried cream (b), and anhydrous milk fat (c); and at day 8 for butter (d), freeze-dried cream (e), and anhydrous milk fat (f). Samples were stained with Nile red and FITC. The black shadow on the image background represents the crystal network of the samples. The yellowish/ brownish area is the liquid fat of the system, and the green areas are protein/water phases. The scale bar indicates $20 \mu \mathrm{m}$
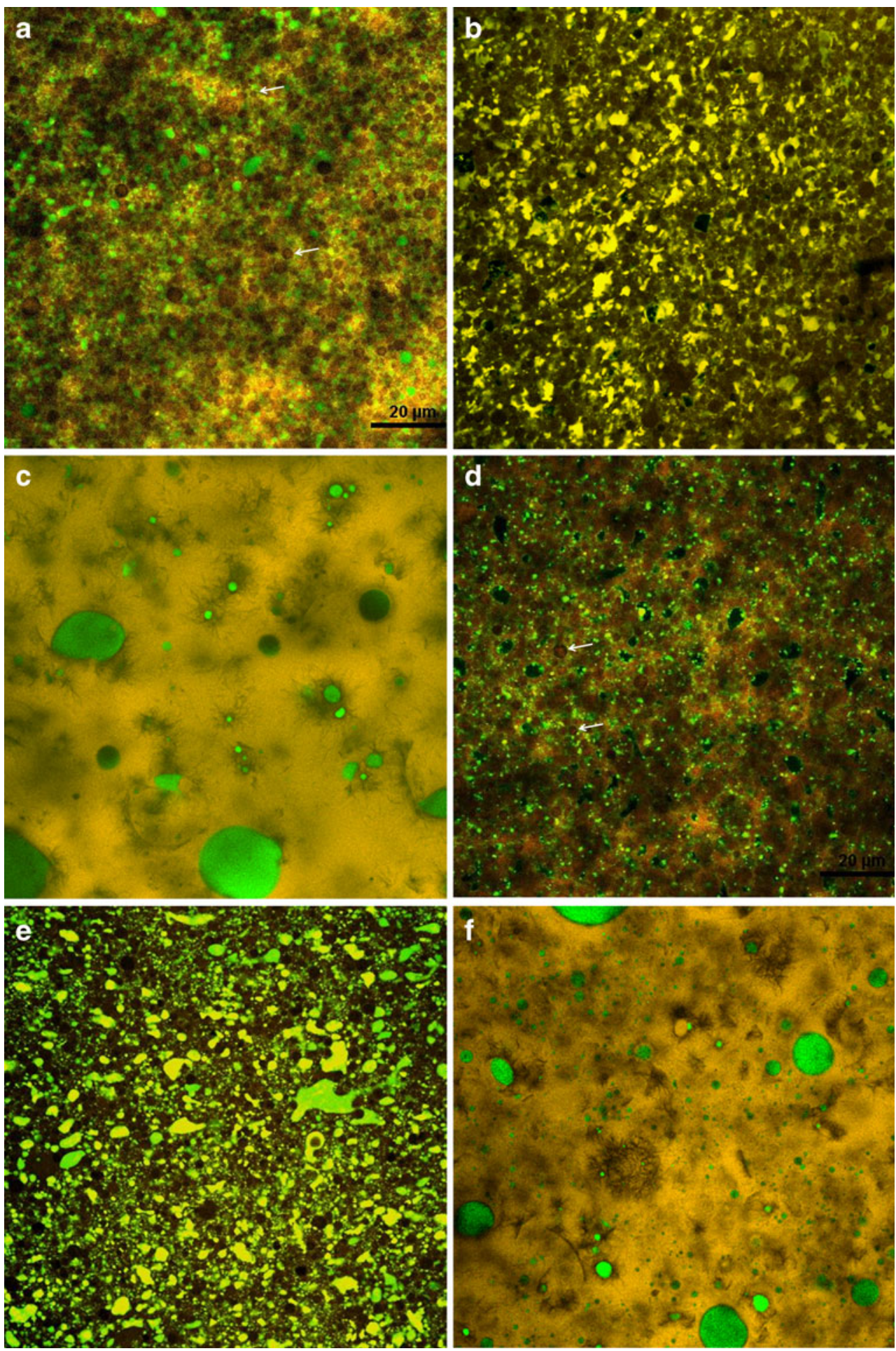

network were visible in the images. Whereas, in the AMF-based emulsion, spherulites were dispersed in liquid oil together with water droplets (Fig. 1c). AMF-based emulsion had the highest $\mathrm{G}^{\prime}$ (Fig. 3), relative hardness as measured by needle geometry (Fig. 4a), and true hardness as measured by penetration test performed with cylinder geometry (Fig. 5a). This behavior was further confirmed by the stress at fracture measurements, both by compression and penetration test (Figs. 5b and 6). Whereas, the relative hardness of AMF-based emulsion, as measured by cone geometry, had the same value as butter (Fig. 4b). Concerning brittleness, only AMF-based emulsion revealed brittle behavior in both penetration test with cylinder geometry, and by compression test, as evaluated by the irregularities showed in the forcedistance curves (Figs. 7 and 8a). AMF-based emulsion and butter had identical elasticity (Fig. 9). On the contrary to AMFbased emulsion, freeze-dried cream had the lowest 
Fig. 2 Confocal laser scanning microscopy images at day 0 for butter (a) and freeze-dried cream (b); and at day 8 for butter (c) and freeze-dried cream (d). Samples were stained with Rhodamine. The yellowish area represents the milk fat protein membrane around the milk fat globules, which are indicated by the black area. The scale bar indicates $20 \mu \mathrm{m}$
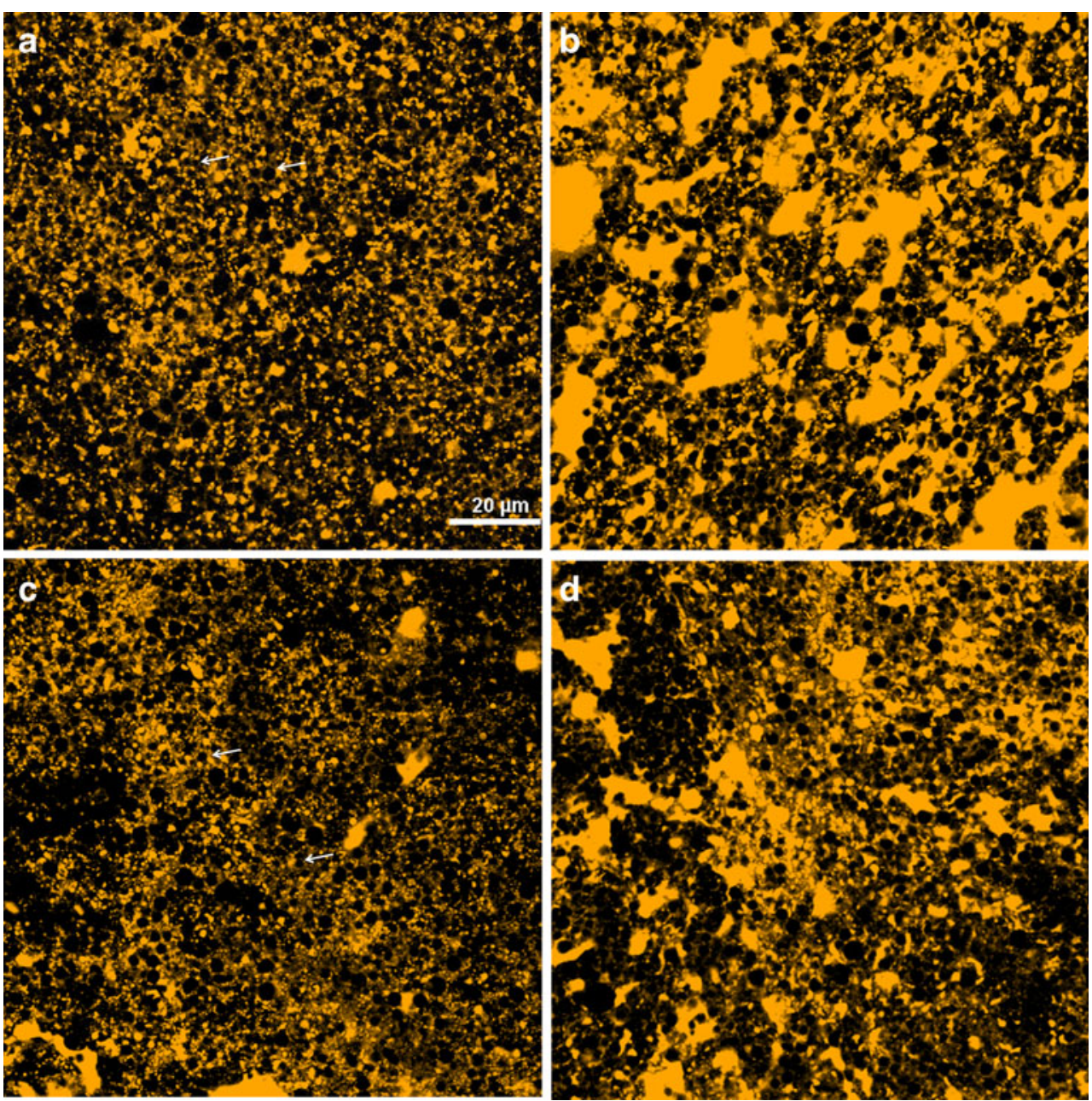

relative hardness as measured by cone geometry (Fig. 4b), and true hardness as measured by penetration test performed with a cylinder geometry (Fig. 5a). This behavior was further confirmed by the stress at fracture measurements (Figs. 5b and 6). Whereas, the $\mathrm{G}^{\prime}$, and the relative hardness as measured by needle had the same value as butter (Figs. 3 and 4a). Concerning elasticity, freeze-dried cream was the most elastic system, as shown by the smaller slope (Fig. 9).

Microstructure and Material Properties of the Milk Fat Systems During Temperature Fluctuations

During temperature fluctuations, butter, the system containing crystallized milk fat both in and outside the milk fat globules, was the one which undergo the major changes in microstructure and material properties. The density of the crystal network in butter, and the amount of flocculated fat globules, significantly increased during temperature fluctuations as shown by the increase in black shadow (Fig. 1d) and black area (mean intensity: $140.1 \pm 3.73$ before temperature fluctuations vs. $156.5 \pm 2.87$ at the end of temperature fluctuations) (Fig. 2c). G' significantly increased for butter at day 5 , and afterwards it was constant through the rest of the period (Fig. 3b). With the longer temperature fluctuations applied at day 4, butter significantly increased in relative hardness as measured by needle, and in true hardness, whereas it decreased in elasticity (Figs. 4a, 5a and 9). The relative hardness of butter, when tested by cone geometry, was significantly higher at the end of the temperature fluctuations (day 6 and day 8) than at day 0 (Fig. 4b). Concerning the stress at fracture, butter had a constant stress up to day 4 for penetration test, and up to day 2 for compression test, afterwards it increases for both tests (Figs. 5b and 6). Butter became brittle only after several temperature fluctuations, when analyzed by penetration test (Fig. 8b). On the contrary, the compression test did not show irregularities in the force-distance curves, at any time of the temperature fluctuations (Fig. 7). AMF-based emulsion showed a different behavior than butter during temperature fluctuations. The microstructure of AMF had few spherulites and a finer network with more contact points than the initial one (Fig. 1f). G', relative hardness as measured by cone, stress at fracture as measured by both tests (except for day 2), and brittleness, 

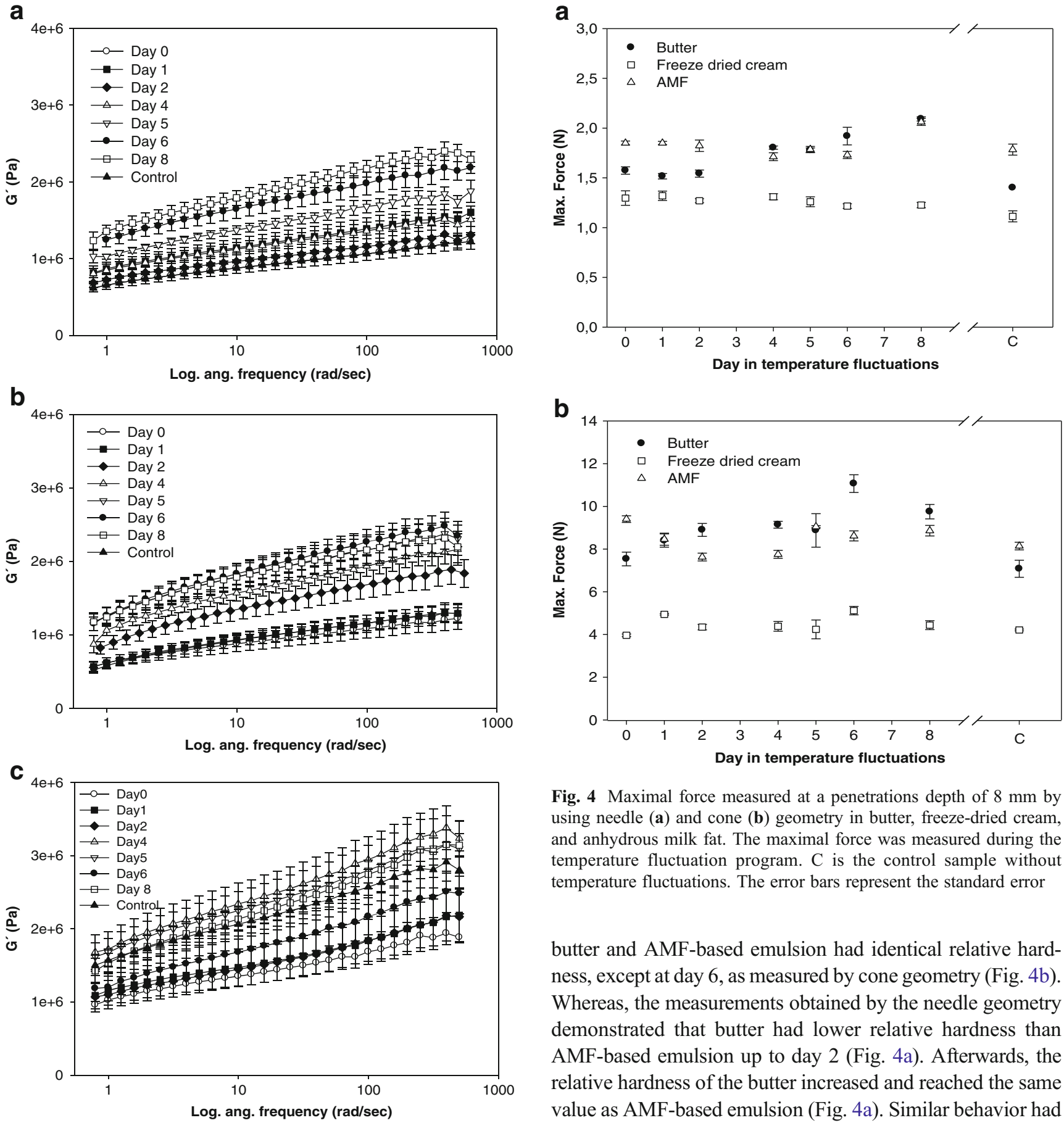

Fig. 3 Elastic modulus $\left(\mathrm{G}^{\prime}\right)$ of freeze-dried cream (a), butter (b), and anhydrous milk fat (c), as a function of angular frequency during a strain of 5.04E-3\%. The control indicates the sample without temperature fluctuations. The error bars represent the standard error

were constant during temperature fluctuations (Figs. 3c, 4b, 5b, 6, 7 and 8a). Whereas, the true hardness increased after temperature fluctuations (Fig. 5a). The relative hardness, as tested by needle geometry, was significantly higher at the end of the temperature fluctuations, day 8 (Fig. 4a). The elasticity decreased at day 5 (Fig. 9). During temperature fluctuations,

Fig. 4 Maximal force measured at a penetrations depth of $8 \mathrm{~mm}$ by using needle (a) and cone (b) geometry in butter, freeze-dried cream, and anhydrous milk fat. The maximal force was measured during the temperature fluctuation program. $\mathrm{C}$ is the control sample without temperature fluctuations. The error bars represent the standard error

butter and AMF-based emulsion had identical relative hardness, except at day 6 , as measured by cone geometry (Fig. 4b). Whereas, the measurements obtained by the needle geometry demonstrated that butter had lower relative hardness than AMF-based emulsion up to day 2 (Fig. 4a). Afterwards, the relative hardness of the butter increased and reached the same value as AMF-based emulsion (Fig. 4a). Similar behavior had the true hardness of butter, which was lower than AMF-based emulsion for day 0 and day 1 , and afterwards it started increasing and reached higher values than AMF-based emulsion (Fig. 5a). The stress at fracture of butter reached significantly higher value than AMF-based emulsion at day 4 for compression test (Fig. 6), whereas for penetration test it only reached significant higher value than AMF-based emulsion at day 8 (Fig. 5b).

In general, temperature fluctuations did not affect the rheological behavior and the material properties of freeze-dried cream. In freeze-dried cream, some of the fat globules also 
a
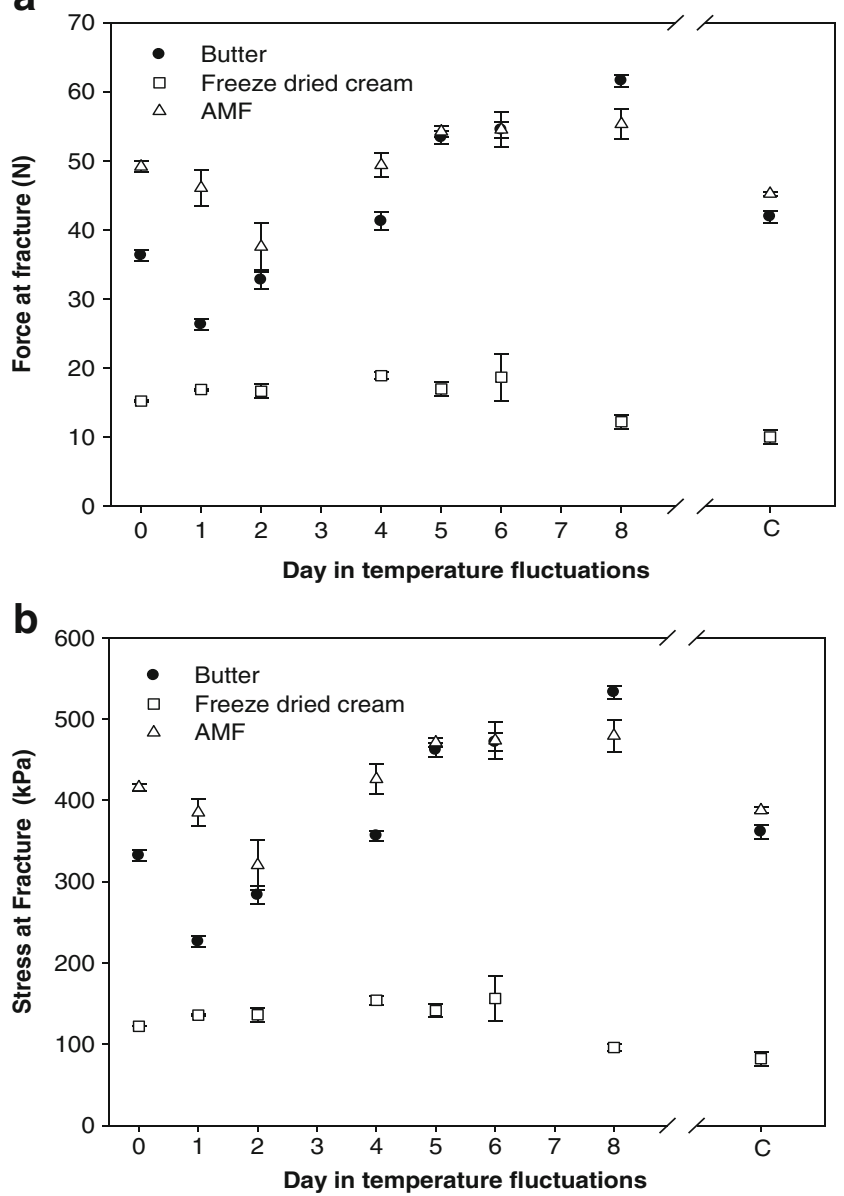

Fig. 5 Force at fracture (a), and stress at fracture (b) obtained by cylinder geometry at a penetration depth of $8 \mathrm{~mm}$ in butter, freeze-dried cream, and anhydrous milk fat. The measurements were performed during the temperature fluctuation program. $\mathrm{C}$ is the control sample without temperature fluctuations. The error bars represent the standard error

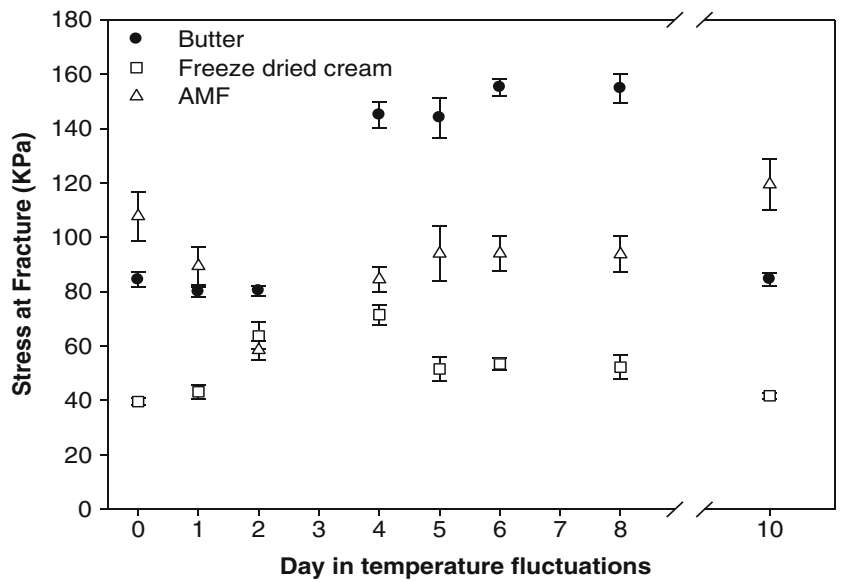

Fig. 6 Stress at fracture obtained by compression test in butter, freeze-dried cream, and anhydrous milk fat. The measurements were performed during the temperature fluctuation program. $\mathrm{C}$ is the control sample without temperature fluctuations. The error bars represent the standard error

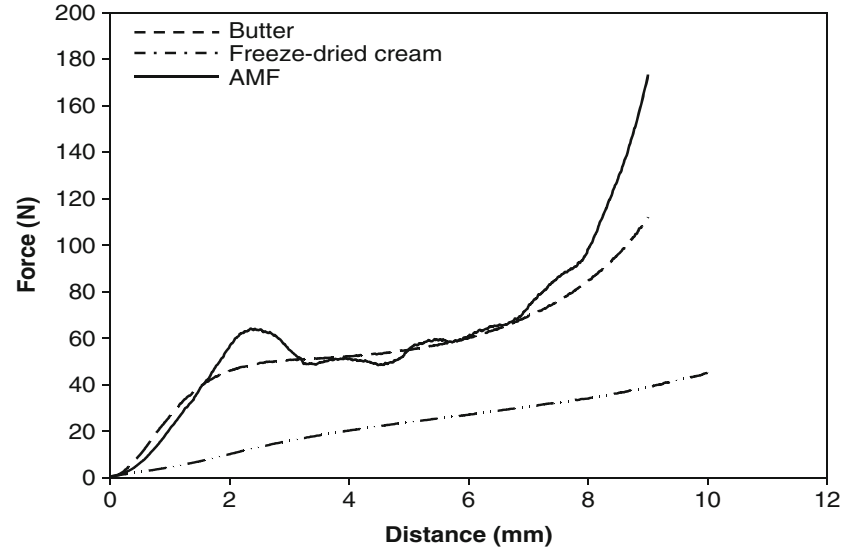

Fig. 7 Example of Force-distance curves obtained by compression test at day 0 for butter, freeze-dried cream, and anhydrous milk fat
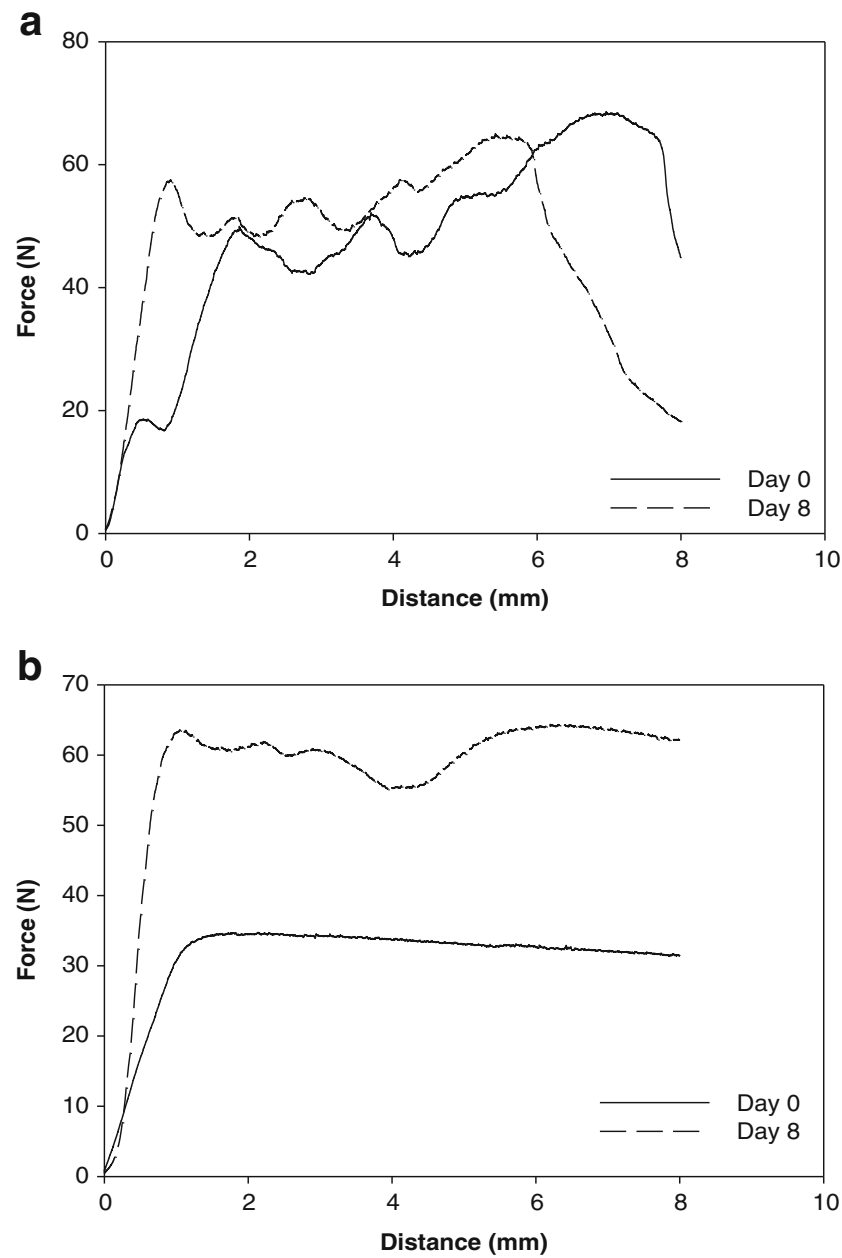

Fig. 8 Example of Force-distance curves obtained by penetration test with cylinder geometry in anhydrous milk fat (a), and in butter (b) analyzed at day 0 and at day 8 during the temperature fluctuation program 


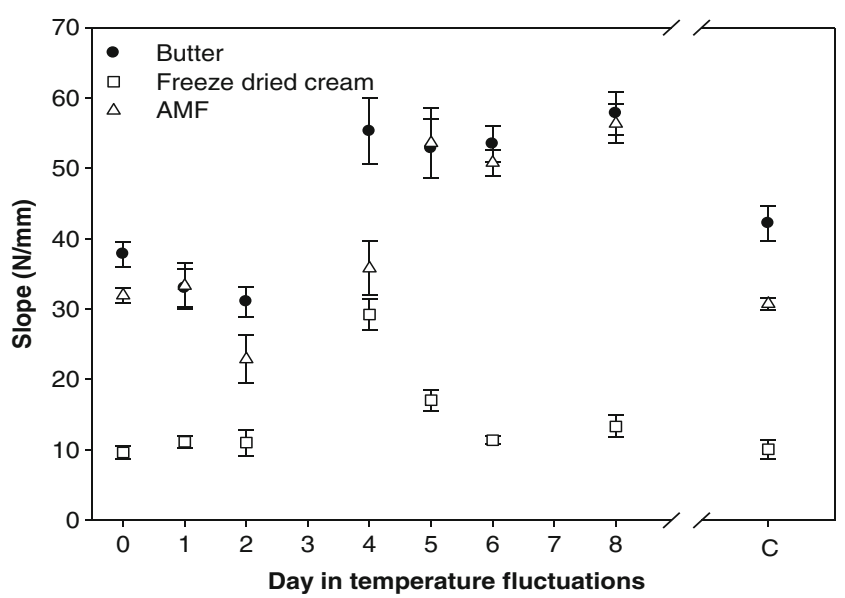

Fig. 9 Maximum slope before sample fractures obtained by compression test in butter, freeze-dried cream, and anhydrous milk fat. The slope was measured during the temperature fluctuation program. $\mathrm{C}$ is the control sample without temperature fluctuations. The error bars represent the standard error

flocculate upon temperature fluctuations (Figs. 1e and 2d). This was further confirmed by the image analysis where a significant increase in black area was reported after temperature fluctuations (mean intensity: $125.9 \pm 4.88$ before temperature fluctuations vs. $144.0 \pm 3.30$ at the end of temperature fluctuations). The relative and true hardness, the stress at fracture (except at day 2 and 4 when measured by compression test), and the elasticity (except at day 4), were constant during temperature fluctuations (Figs. 4, 5, 6 and 9). The $\mathrm{G}^{\prime}$ was significantly higher only at day 8 , when compared to day 0 (Fig. 3a). Freeze-dried cream did not show brittleness (Fig. 7).

\section{Melting Behavior and Level of SFC of the Milk Fat Systems}

Milk fat is characterized by an extreme diversity of fatty acids; hence triacylglycerols (TAGs) composition, which induces to a broad thermal range, from $-40{ }^{\circ} \mathrm{C}$ to $40{ }^{\circ} \mathrm{C}$. The melting profile of milk fat is characterized by three melting fractions. The low melting fraction (LMF) is below $10^{\circ} \mathrm{C}$, the medium melting fraction (MMF) from $10{ }^{\circ} \mathrm{C}$ to $19{ }^{\circ} \mathrm{C}$ and the high melting fraction (HMF) above $20{ }^{\circ} \mathrm{C}$ [11].

Butter and freeze-dried cream had the same melting profile with two melting fractions, the MMF and the HMF (Fig. 10). No changes in melting profile took place from day 0 to day 8 (data not shown). The thermogram of AMF-based emulsion had a third fraction, which was in the range of the HMF, above $20{ }^{\circ} \mathrm{C}$, however the temperature of the minimum peak was lower than the one of the HMF common with the other systems (Fig. 10).

Butter and freeze-dried cream had the same SFC at day 0 and it significantly decreased after temperature fluctuations (Table 2). AMF-based emulsion had lower level of SFC when compared to butter and freeze-dried cream at day 0. Furthermore, SFC for AMF-based emulsion was not affected by

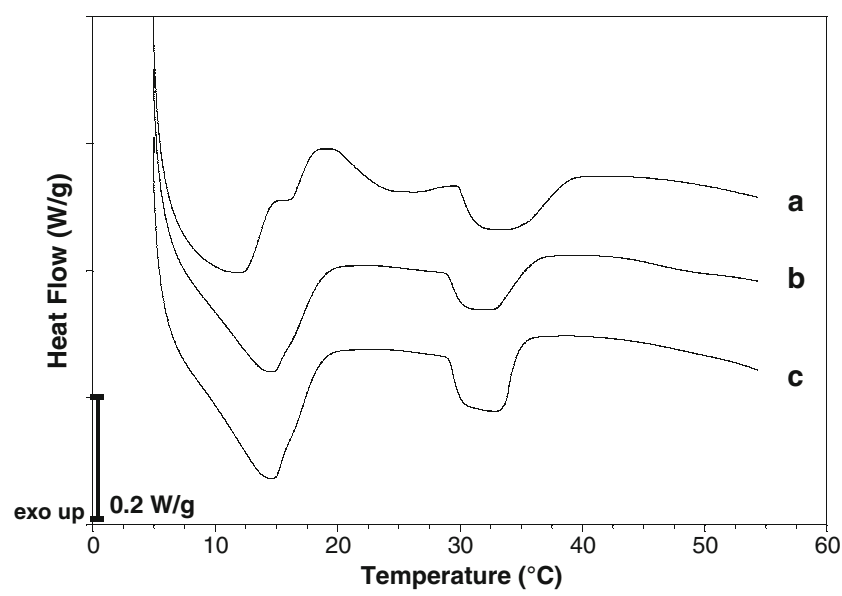

Fig. 10 Example of thermograms at day 0 of anhydrous milk fat (a), butter (b), and freeze-dried cream (c). The heating rate was $5{ }^{\circ} \mathrm{C} / \mathrm{min}$

temperature fluctuations (Table 2). Despite the statistical test indicates differences in SFC, this was in a small range which was not considered to cause major changes in the material properties of the milk fat systems.

\section{Discussion}

Microstructure and Material Properties of the Milk Fat Systems Before Temperature Fluctuations

The three systems evaluated in this study all contained milk fat with nearly similar SFC $48-52 \%$ at $5{ }^{\circ} \mathrm{C}$, despite this, the material properties were different among them. Butter is a W/O emulsion with a crystalline fat network both in the continuous phase and inside the milk fat globules. In contrasts to butter, we produced a system with concentrated intact milk fat globules by freeze drying cream, hereby all the crystallized fat will be inside the globules. The dry matter content of the two systems was similar. However, in interpretation of the data it has to be taken into account that the protein content was higher in the freeze-dried cream, although the contribution to the material properties from milk proteins in these systems is estimated to be low. The melting behavior of the three systems indicates that the fatty

Table 2 Solid fat content (SFC) at day 0 and day 8 for butter, freezedried cream and anhydrous milk fat (AMF)

\begin{tabular}{lcl}
\hline Sample & Day 0 & Day 8 \\
\hline Butter & $50.35 \pm 0.24$ & $48.98 \pm 0.18^{\mathrm{a}}$ \\
Freeze-dried cream & $51.53 \pm 0.33$ & $49.76 \pm 0.25^{\mathrm{a}}$ \\
AMF & $47.57 \pm 0.56^{\mathrm{b}}$ & $48.24 \pm 0.44$ \\
\hline
\end{tabular}

${ }^{\text {a }}$ significant effect of time

${ }^{\mathrm{b}}$ significant effect of system 
acids composition of the milk fat used in the three systems was rather similar. The extra melting fraction obtained in AMF-based emulsion, and not present in the thermograms of butter and freeze-dried cream, could be explained by the presence of fat globules interfering with the weak signal of the TAGs included in that fraction. In general, freeze-dried cream had a less stiff, softer, and more elastic structure than butter and AMF-based emulsion, with the latter having the most stiff and hardest structure. Among AMF-based emulsion samples, the rheological properties can be different even though the SFC level is similar, which is due to different microstructure of the crystalline network [12]. Variations in size of milk fat crystal clusters can be obtained by manipulating cooling rate or by applying shear $[13,14]$. In the present study, the large variation in material properties was achieved by changing the continuous phase or by increasing the number of intact milk fat globules of the milk fat system. When a continuous crystal network was present, as in butter, the system was approximately two times harder, and slightly stiffer than freeze-dried cream in which the crystals or crystal clusters were not connected. AMF-based emulsion was two times stiffer and one time harder than butter despite having bigger crystals (approximately $20 \mu \mathrm{m}$ ), thus less contact points. In general, milk fat crystals in emulsion are smaller than crystals in AMF-based emulsion. Smaller crystals lead to a crystal network with more contact points, which in turn results in a material with higher stiffness than a material with less contact points [13]. However, larger crystals can be characterized by stronger connections between each other, thus by a higher elastic modulus than systems with smaller crystals and weaker connections $[12,15,16]$. Therefore, both particle size and nature of the interactions between crystals should be considered when evaluating material properties. AMF-based emulsion had higher stiffness than butter, which indicates stronger secondary bonds between crystals clusters; moreover, milk fat globules did not interrupt the crystal network. Presence of milk fat globules contributes to a decrease in hardness and stiffness of the milk fat system. This is due to the presence of milk fat crystals inside the milk fat globules, which do not contribute to the continuous crystal network as less milk fat is available to crystallize outside the milk fat globules, therefore a less firm and less hard structure is formed. Moreover, the presence of fat globules interferes with the continuous crystal network by disturbing the continuity of the network. The response of the material properties to presence of milk fat globules contributed also to the elasticity and brittleness of the systems, beside stiffness and hardness. Freeze-dried cream, despite the softness of the system, was approximately three times more elastic than butter and than AMF-based emulsion, in addition it did not show brittleness (Figs. 9 and 7). AMF-based emulsion and butter had identical elasticity (Fig. 9), but different brittleness (Figs. 7 and 8), arguing for the contribution of presence of intact milk fat globules and volume fraction of the fat crystal network in the continuous phase. The milk fat globules led to high deformability of the material before fracture occurred. Whereas, the volume fracture of the crystal network in the continuous phase has an opposite effect as it contributes to a system more prone to fracture. Therefore, by increasing the amount of intact milk fat globules elasticity increases and brittleness decreases. The opposite occurred by increasing the volume fraction of the crystal network in the continuous phase, thus by decreasing the amount of fat globules. The differences in material properties were independent from the level of SFC and melting behavior, as no differences were reported by comparing butter and freeze-dried cream.

\section{Effect of Temperature Fluctuations on Microstructure and Material Properties of the Milk Fat Systems}

Temperature fluctuations in butter led to an increase in stiffness, relative and true hardness, stress at fracture, brittleness and to a decrease in elasticity. Melting and recrystallization of butter caused milk fat globules to flocculate (Fig. 2a and c). This seems to structure a denser and stronger continuous crystallized network with less single interference from intact milk fat globules. Similar finding were reported after tempering treatment of whipped dairy cream and of foam systems, which leads to an increase in stiffness as consequence of partial coalescence phenomena $[17,18]$. Partial coalescence phenomenon was not likely to occur in the studied systems, as air was not the main component of the microstructure. Presence of air in the system, such as in whipped dairy cream and foam, promotes partial coalescence as milk fat globules locate at the air/water interface, there flocculation and partial coalescence of fat globules occur $[4,19,20]$. In addition, sintering, which refers to the formation of solid bridges between crystals and crystal clusters, may also be responsible for the denser and stronger crystallized network [21]. The solid bridges formed by sintering are stronger than Van der Waals bonds, which connect fat crystals in the continuous network. Thus, the structure will be firmer and more stable after temperature fluctuations. The prolonged exposure to room temperature of butter at day 4 , caused a significant increase in relative and true hardness, and a decrease in elasticity (Figs. 4a, 5a and 9). This argues for the higher level of melting to cause more changes to the microstructure, i.e. flocculation of more milk fat globules, and an even denser and stronger continuous network was formed during the following recrystallization. No changes in material properties were observed when the systems were further stored at $5{ }^{\circ} \mathrm{C}$ for 2 days (day 8). AMF-based emulsion increased in relative and true hardness, and decreased in elasticity after the temperature 
fluctuation program (Figs. 4b, 5a and 9). The CLSM images showed a microstructure composed of smaller crystals in a denser network after temperature fluctuation program. This is quite similar to the observations for the continuous fat crystal network of butter. Melting followed by recrystallization is speculated to increase homogeneous crystals at the expense of mixed crystals which will result in larger crystals [3]. The present data from DSC did not demonstrate such changes in any of the fat systems. However, temperature fluctuations might have caused the melting of some of the MMF TAGs of the crystal clusters, which upon cooling and storage recrystallized differently from the original form. This could have caused more binding between neighbor crystals, or formation of sintered bonds, thus a denser crystal network which in turn led to a harder and less elastic system. During temperature fluctuations, butter reached higher values than AMFbased emulsion in true hardness and stress at fracture, whereas relative hardness and elasticity were equal (Figs. 5, 6, 4 and 9). The denser and stronger network formed during temperature fluctuations in butter contributed to the increase in hardness and brittleness, and to a decrease in elasticity.

Emulsion instability was avoided or minimum in freezedried cream, as its material properties were constant during temperature fluctuation program, indicating that melting and recrystallization of milk fat inside intact fat globules are not affecting the material properties. This fosters the speculation that producing butter with more intact fat globules would result in a product which is more resistant to temperature fluctuations. The changes in material properties obtained after temperature fluctuations further confirmed the hypothesis that crystallization occurring within the milk fat globules does not affect the rheological and material properties of the system. However, flocculation of fat globules dispersed in a continuous fat phase leads to a different spatial configuration of the microstructural elements, therefore to a denser crystal network in the continuous phase. This endorses the hypothesis that by increasing the volume fraction of the continuous crystal network the elasticity decreases, while the brittleness increases.

During temperature fluctuations the milk fat systems studied were stable against physical emulsion destabilization, such as phase separation due to coalescence of water droplets. Freeze-dried cream was most likely stabilized by the emulsifiers naturally present in the milk fat globule membrane. Butter was mainly stabilized by the continuous crystal network. Whereas, in AMF-based emulsion pickering stabilization, which refers to the presence of surface active crystals at the interface between water and oil, was observed. Similar findings were previously observed in butter and margarine [22-24].

\section{Relations Between Rheological Techniques}

In butter and in AMF the relative hardness measurements, obtained by large deformation rheology, had similar trend of the stiffness measurements obtained by small deformation rheology. Whereas, butter and freeze-dried cream had identical stiffness but different hardness. This can be argued by the different emulsion type, thus continuous phase in the system, and by the amount of fat globules which can both affect the hardness of the system. However, no significant differences were measured in the stiffness of the systems, which is assumed to be based on the strength of the secondary bonds. Narine and Marangoni [25] showed that in pure fat systems an increase in elastic modulus is followed by an increase in hardness, as measured by the standardized cone penetrometry method [26]. Thus, the elastic modulus can be used as an indicator of hardness only when studying pure fat systems or fat system with a fat-based continuous phase. Moreover, different geometries and rheological methods give different hardness value. The cone geometry is widely used in the fat and oil industry for the measurement of the consistency and hardness of plastic fats [26]. However, it tends to cause fractures in the material due to an increase in cross sectional area during penetration into the samples [27]. The needle geometry is more suitable for hard fat, as fully hydrogenated canola oil [27], moreover it is characterized by a constant contact area with the sample. In the present study, needle geometry was more sensitive for hard fat, but it was very suitable for soft fat as well, as shown by testing freeze-dried cream.

Similar trend in stress at fracture was obtained by compression test and by penetration tests with cylinder, despite the different contact areas with the sample and the forces involved during the test. However, the force-distance curves obtained by penetration test showed a clear breaking point in all systems studied, at any temperature fluctuation day (Fig. 8). Whereas, the force-distance curves obtained by parallel plates showed a clear breaking point in AMF-based emulsion and in butter but, no clear breaking point was observed for freeze-dried cream (Fig. 7). This suggested that the penetration test performed with a cylinder is a good indicator of the true hardness, stress at fracture, and also brittleness. The advantage of this geometry compared to cone and needle is that more accurate information can be obtained by the force-distance curve. The needle geometry was more sensitive than the cone geometry to determine relative hardness, as it detected changes in AMF-based emulsion which were not detected by cone, moreover it detected changes in butter already at day 4 .

\section{Conclusion}

Crystallization occurring inside milk fat globules did not contribute to the hardness and stiffness of the system. However, presence of intact milk fat globules in the system conferred a higher elasticity and contributed to a decrease in hardness and stiffness when interfered with the continuous crystal network. The amount and type of connections between crystals and crystal clusters 
contributed to the hardness and stiffness of the system. Temperature fluctuations caused an increase in hardness, stiffness, and brittleness with concomitant decrease in elasticity in systems containing crystals and crystal clusters in the continuous phase, such as butter and AMFbased emulsion. The changes in structure were more drastic when part of the intact fat globules was dispersed in a fat phase, such as in butter, as flocculation led to the formation of a denser and stronger network. However, by increasing the amount of fat globules and decreasing the amount of fat in the continuous phase, a more resistant system to temperature fluctuation is expected. A proper material characterization of milk fat systems is achieved only by studying several material properties and coupling them with rheological tests and microscopy images.

Acknowledgment The authors wish to thank Arla Foods, The Ministry of Food, Agriculture and Fisheries (grant no. 3414-09-02406), The Danish Dairy Research Foundation, and FOOD Denmark for financing the $\mathrm{PhD}$ project. Marianne Hammershøj is acknowledged for the valuable discussions about the rheological data.

\section{References}

1. M. Van den Tempel, J. Colloid Sci. 16, 284 (1961)

2. A.J. Haighton, Fette Seifen, Anstrichm 65, 479 (1963)

3. A.C. Juriaanse, I. Heertje, Food Microstruct. 7, 181 (1988)

4. H. Mulder, P. Walstra, The Milk Fat Globule, (Commonwealth Agricultural Bureaux, Farnham Royal, England 1974)
5. P. Walstra, E.C.H. van Beresteyn, Neth. Milk Dairy J. 29, 35 (1975)

6. C. Lopez, F. Lesieur, P. Bourgaux, G. Keller, M. Ollivon, J. Colloid Interface Sci. 240, 150 (2001)

7. C. Lopez, F. Lavigne, P. Lesieur, G. Keller, M. Ollivon, J. Dairy Sci. 84, 2402 (2001)

8. E. Fredrick, D. Van de Walle, P. Walstra, J.H. Zijtveld, S. Fischer, P. Van der Meeren, K. Dewettinck, Int. Dairy J. 21, 685 (2011)

9. S. Gallier, D. Gragson, R. Jimenez-Flores, D. Everett, J. Agric. Food Chem. 58, 4250 (2010)

10. P. Buldo, L. Wiking, J. Am. Oil Chem. Soc. 89, 787 (2012)

11. E. Deffense, J. Am. Oil Chem. Soc. 70, 1193 (1993)

12. M.L. Herrera, R.W. Hartel, J. Am. Oil Chem. Soc. 77, 1189 (2000)

13. L. Wiking, V. De Graef, M. Rasmussen, K. Dewettinck, Int. Dairy J. 9, 424 (2009)

14. N. Kaufmann, V. De Graef, K. Dewettinck, L. Wiking, Food Biophys. 7, 308 (2012)

15. M.L. Herrera, R.W. Hartel, J. Am. Oil Chem. Soc. 77, 1197 (2000)

16. N. Alberola, C. Bas, P. Mele, C. R. Acad. Sci. II Mécanique, Physique, Chimie, Astronomie 319(1129) (1994)

17. N. Drelon, E. Gravier, L. Daheron, L. Boisserie, A. Omari, F. LealCalderon, Int. Dairy J. 16, 1454 (2006)

18. E. Gravier, N. Drelon, L. Boisserie, A. Omari, F. Leal-Calderon, Colloids Surf. A 282-283(360) (2006)

19. D.F. Darling, J. Dairy Res. 49, 695 (1982)

20. N.E. Hotrum, Ph.D. Thesis, Wageningen University, The Netherlands, (2004)

21. W. Kloek, T. Van Vliet, P. Walstra, J. Texture Stud. 36, 544 (2005)

22. I. Heertje, Food Struct. 12, 77 (1993)

23. D. Rousseau, L. Zilnik, R. Khan, S. Hodge, J. Am. Oil Chem. Soc. 80, 957 (2003)

24. D. Rousseau, S. Ghosh, H. Park, J. Food Sci. E 74, E1 (2009)

25. S.S. Narine, A.G. Marangoni, Lebensm. Wiss. Technol. 34, 33 (2001)

26. AOCS, Official Method Cc 16-60: Consistency Penetrometer Method. (American Oil Chemists' Society, Champaign, IL 1960)

27. M.V. Boodhoo, K.L. Humphrey, S.S. Narine, Int. J. Food Prop. 12, 129 (2009) 